\title{
S1 Code
}

In this supporting information we provide the R-code and JAGS-code used to run the models in the main text. R-packages on which the analysis depends, are included at the start. The code used two pieces of data as provided in S1 Dataset and S2 dataset, being the biomass data and covariate data respectively.

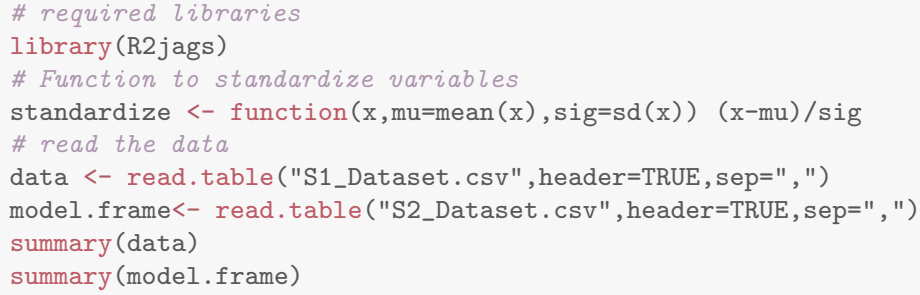

Below, for each of the seven models in Table 3 in the main text, we first create a dataset based on the two loaded datasets, write a JAGS model file, and run the model through JAGS. 


\section{Null model}

Setup the data for running the JAGS model:

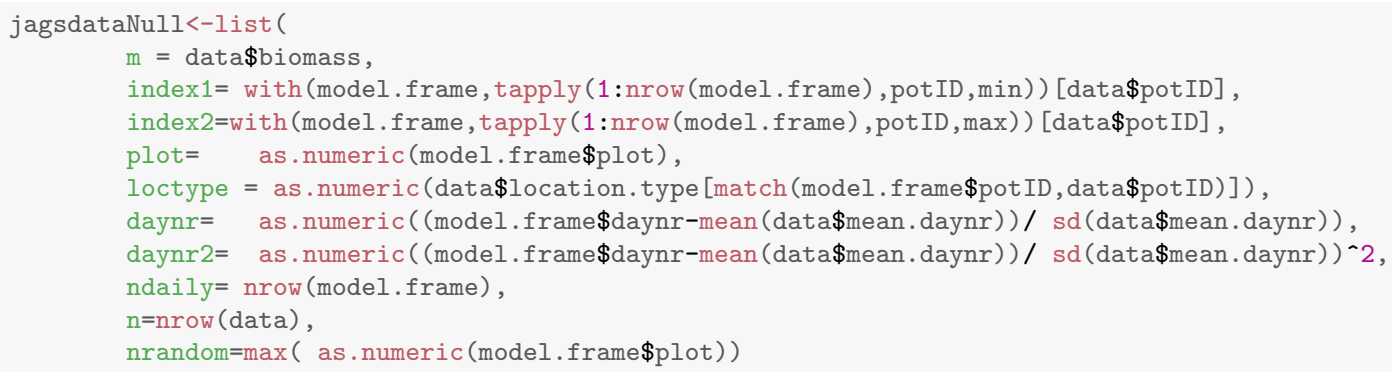

Write JAGS model file:

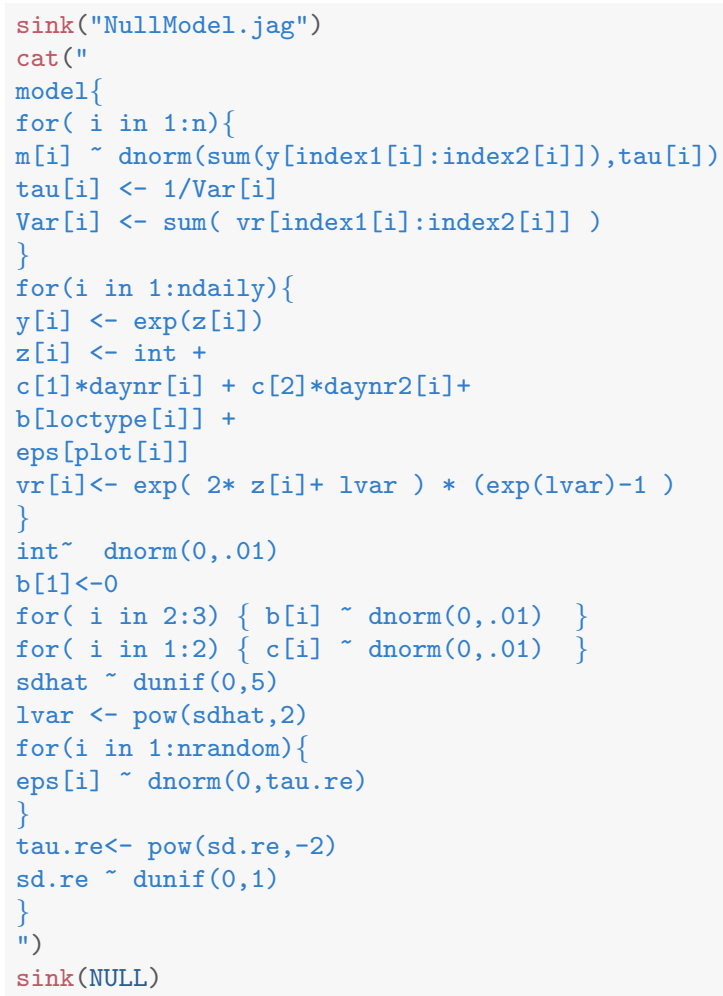

Run the model:

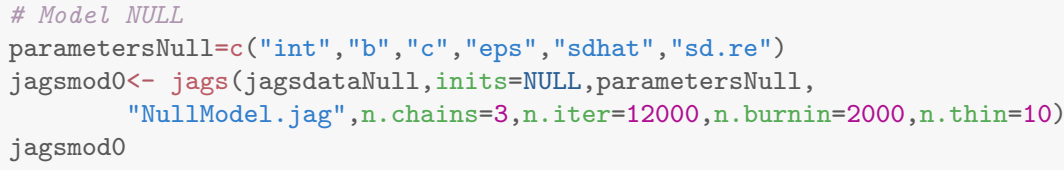




\section{Basic model}

Setup the data for running the JAGS model:

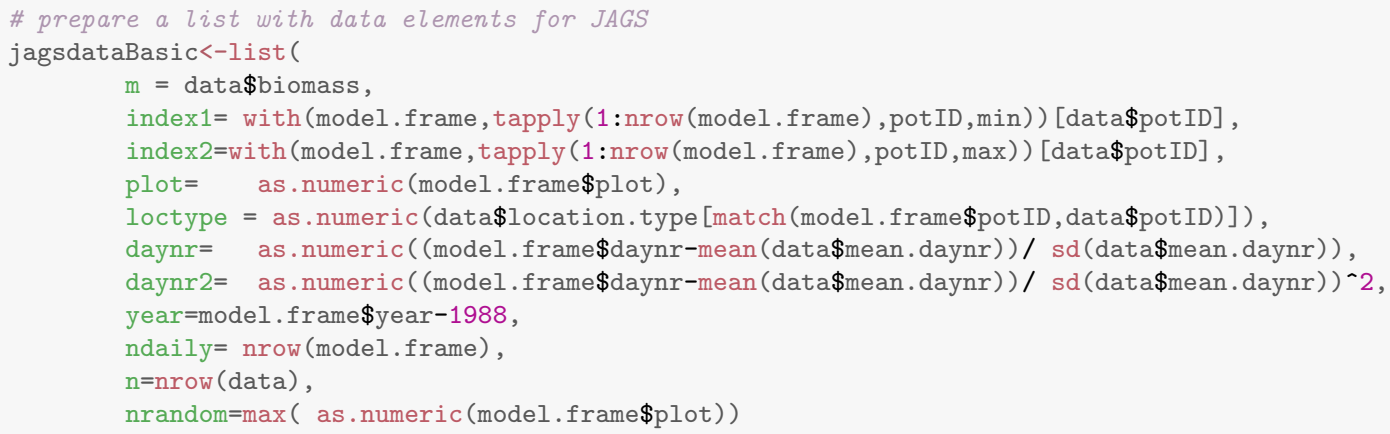

\section{Write JAGS model file:}

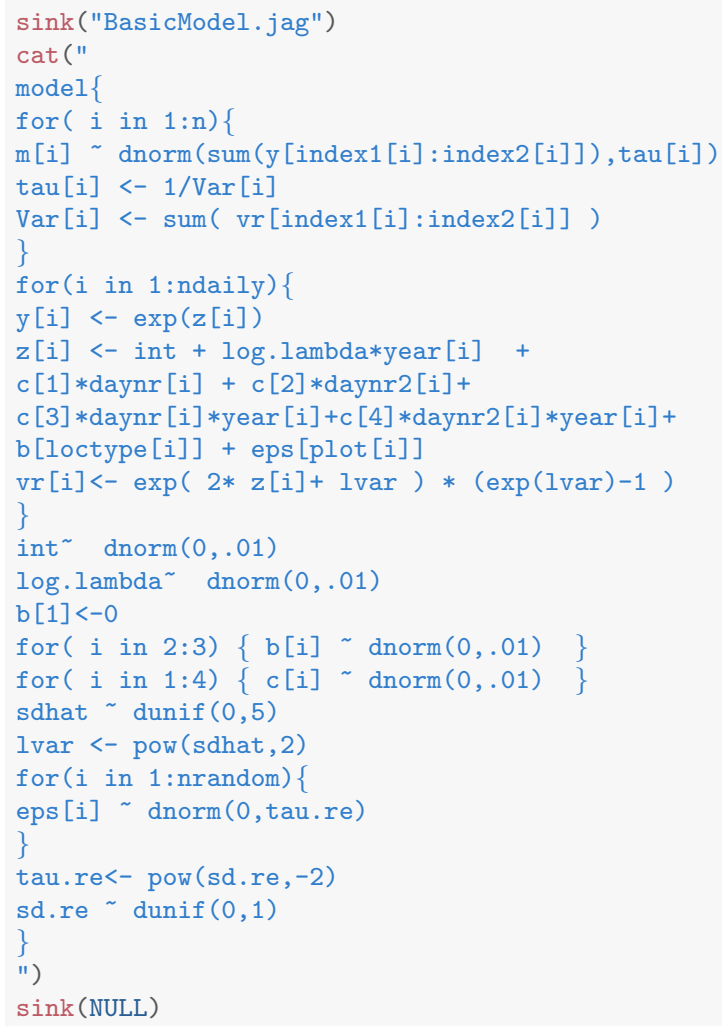

Run the model:

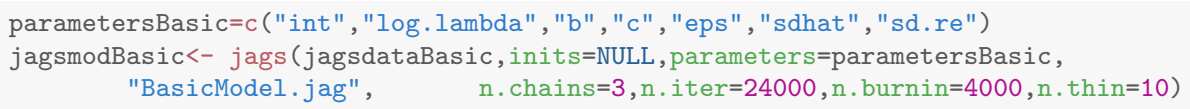




\section{Weather model}

Setup the data for running the JAGS model:

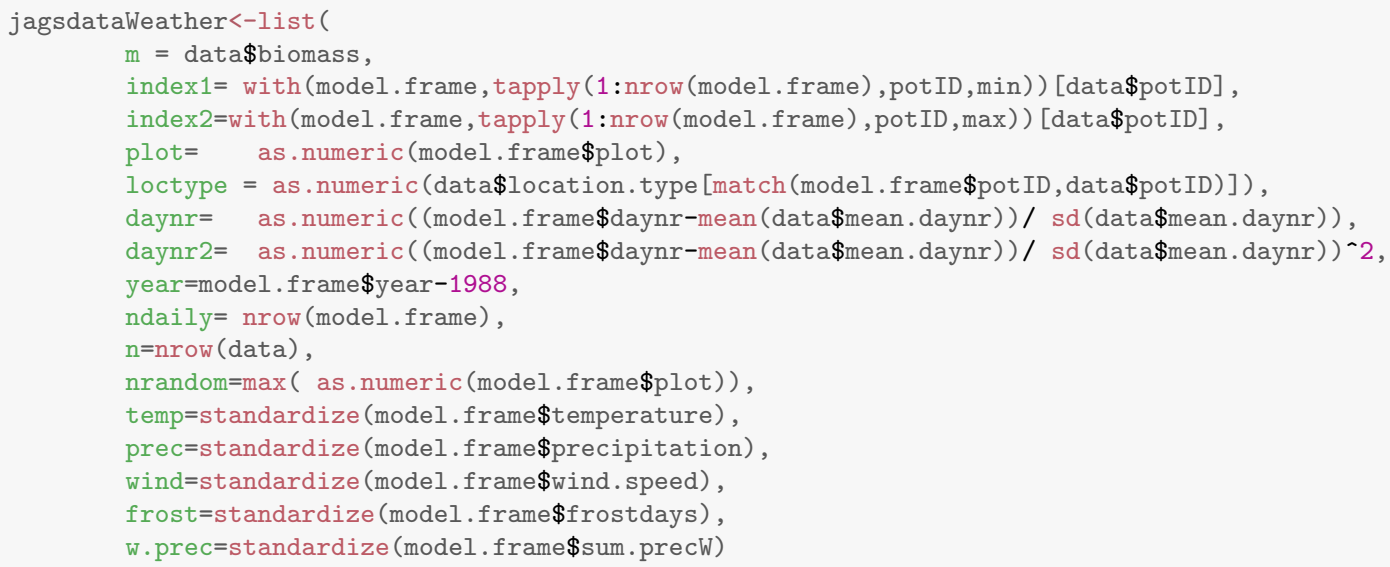

Write JAGS model file:

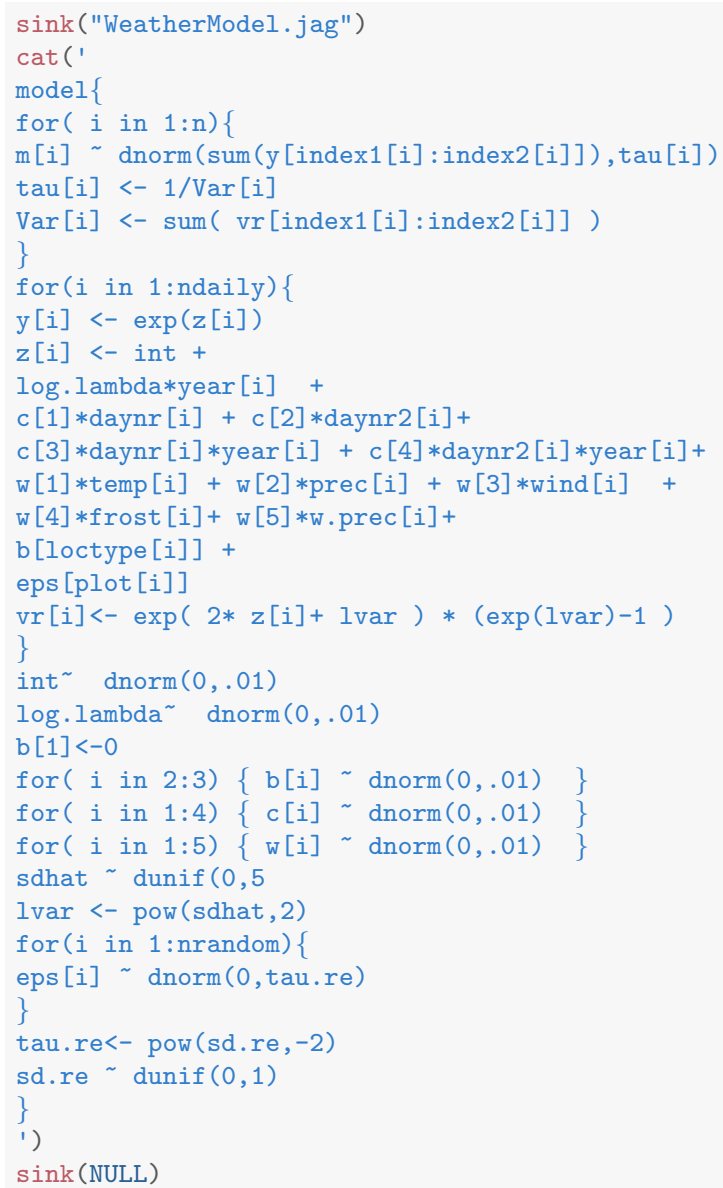

Run the model:

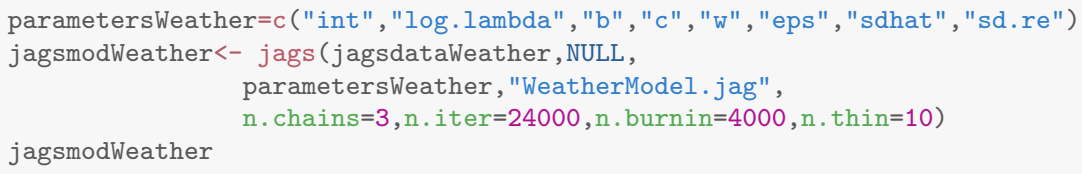




\section{Habitat model}

Setup the data for running the JAGS model:

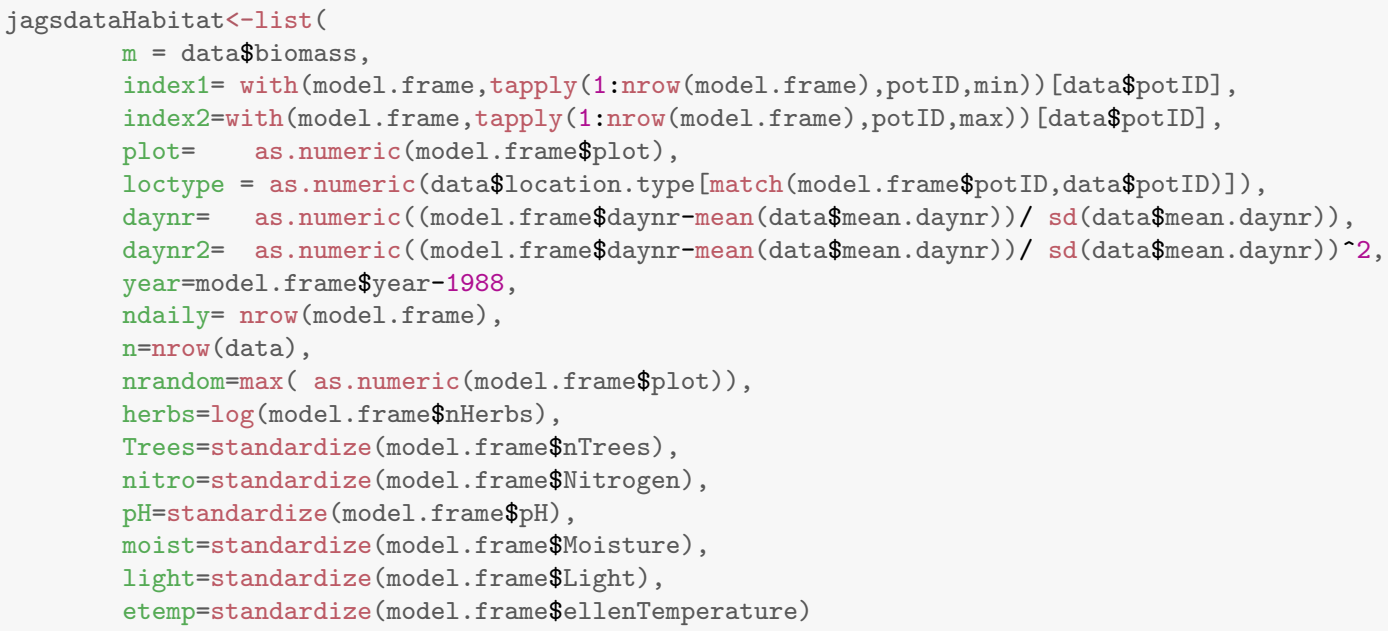

Write JAGS model file:

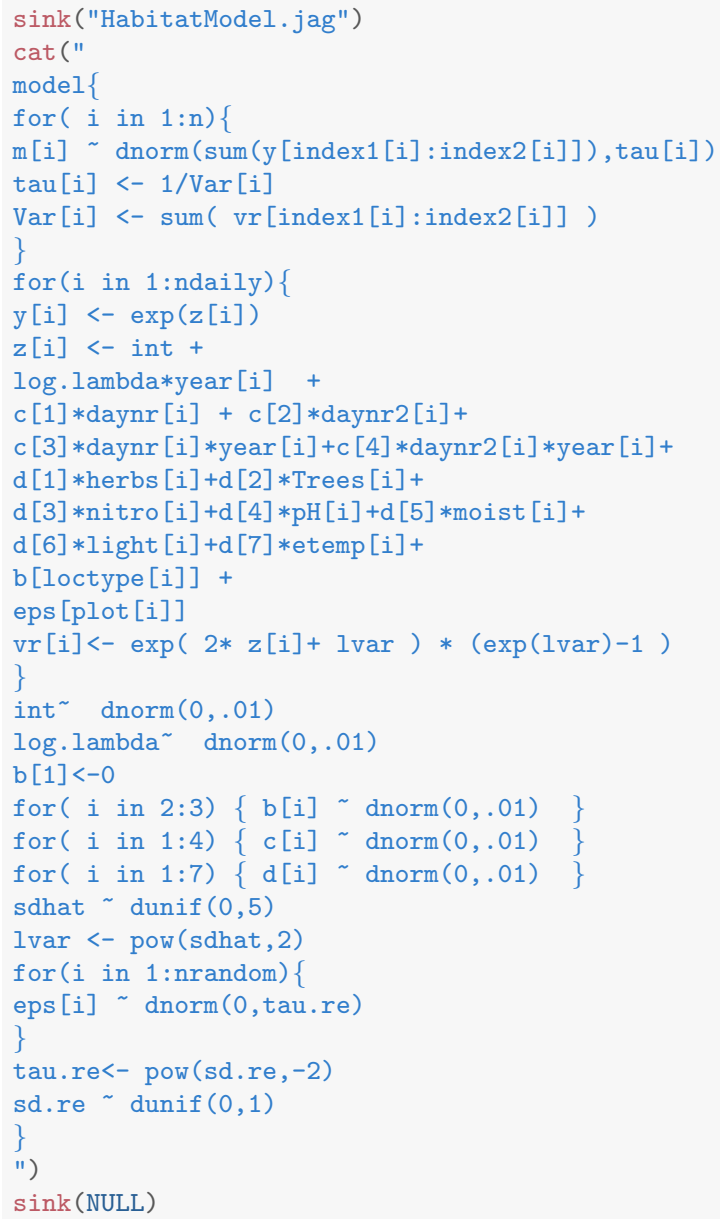

Run the model:

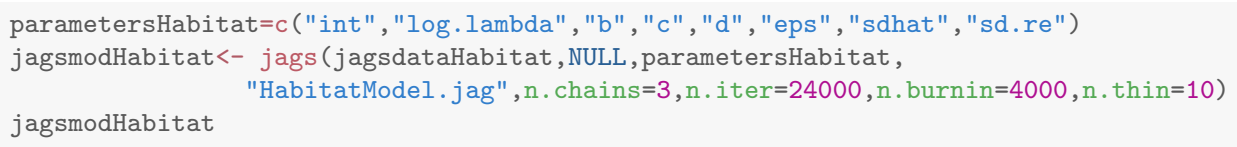




\section{Landuse model}

Setup the data for running the JAGS model:

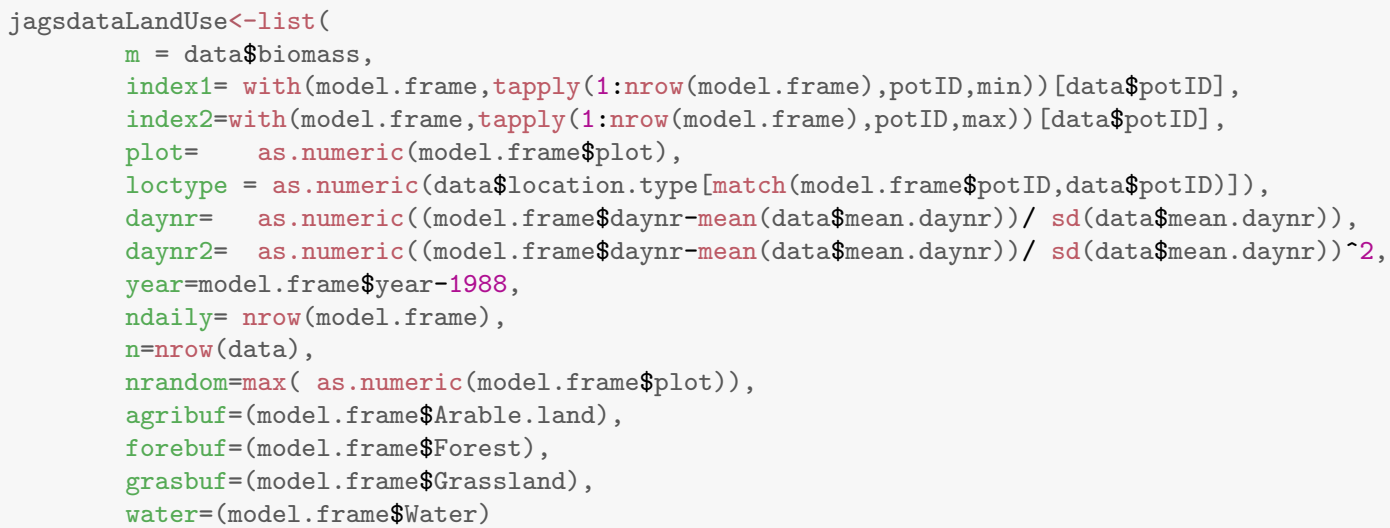

Run the model:

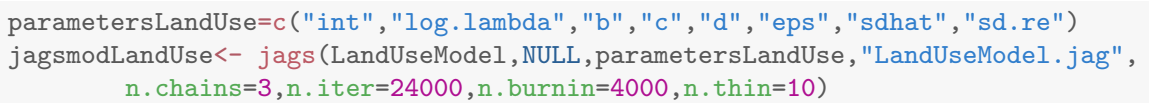




\section{Landuse with interactions model}

Setup the data for running the JAGS model:

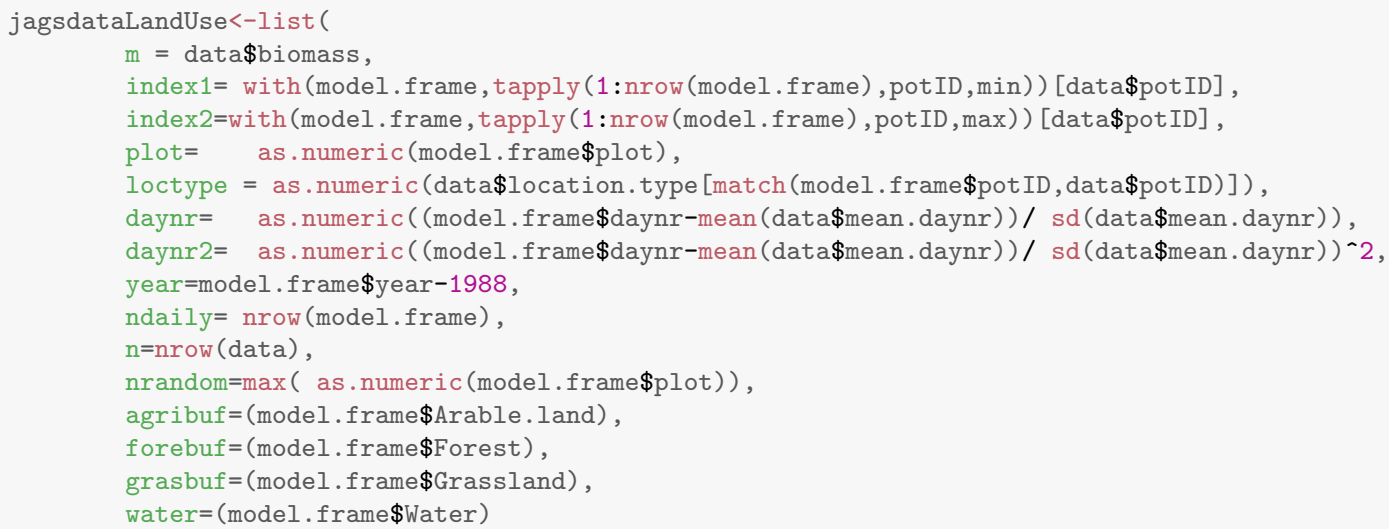

Write JAGS model file:

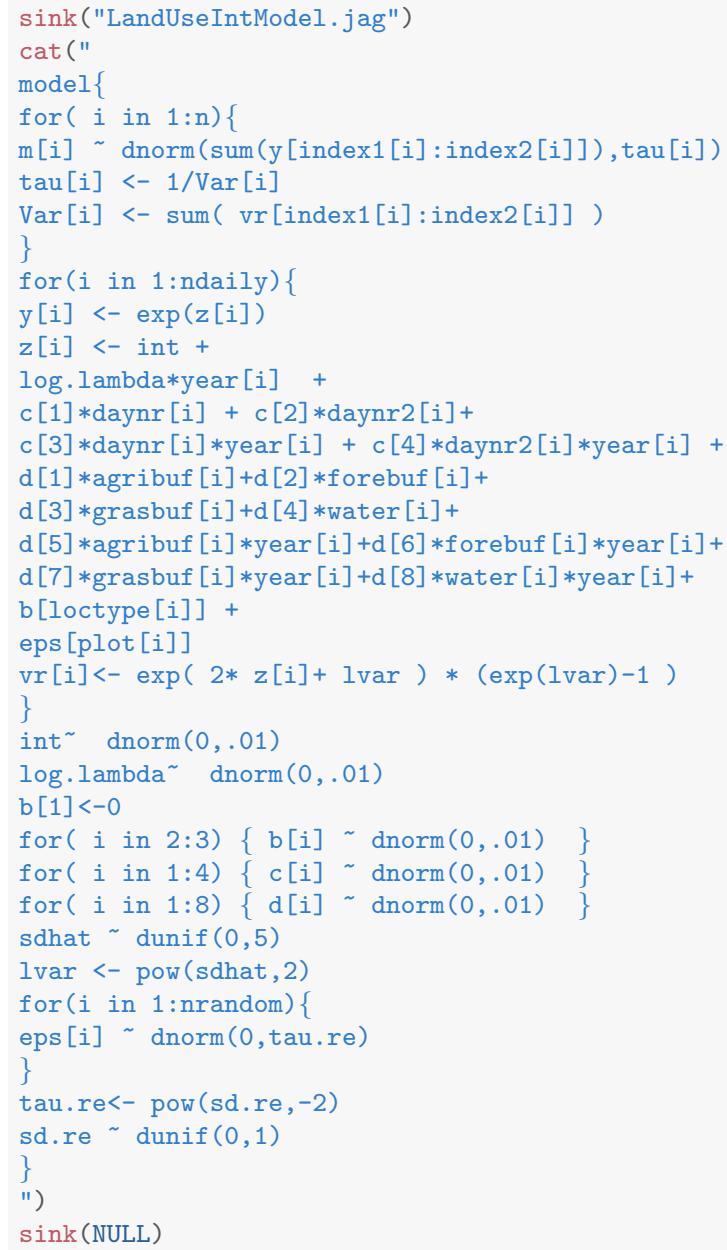

Run the model:

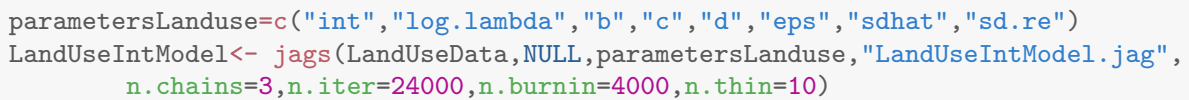




\section{Full model}

Setup the data for running the JAGS model:

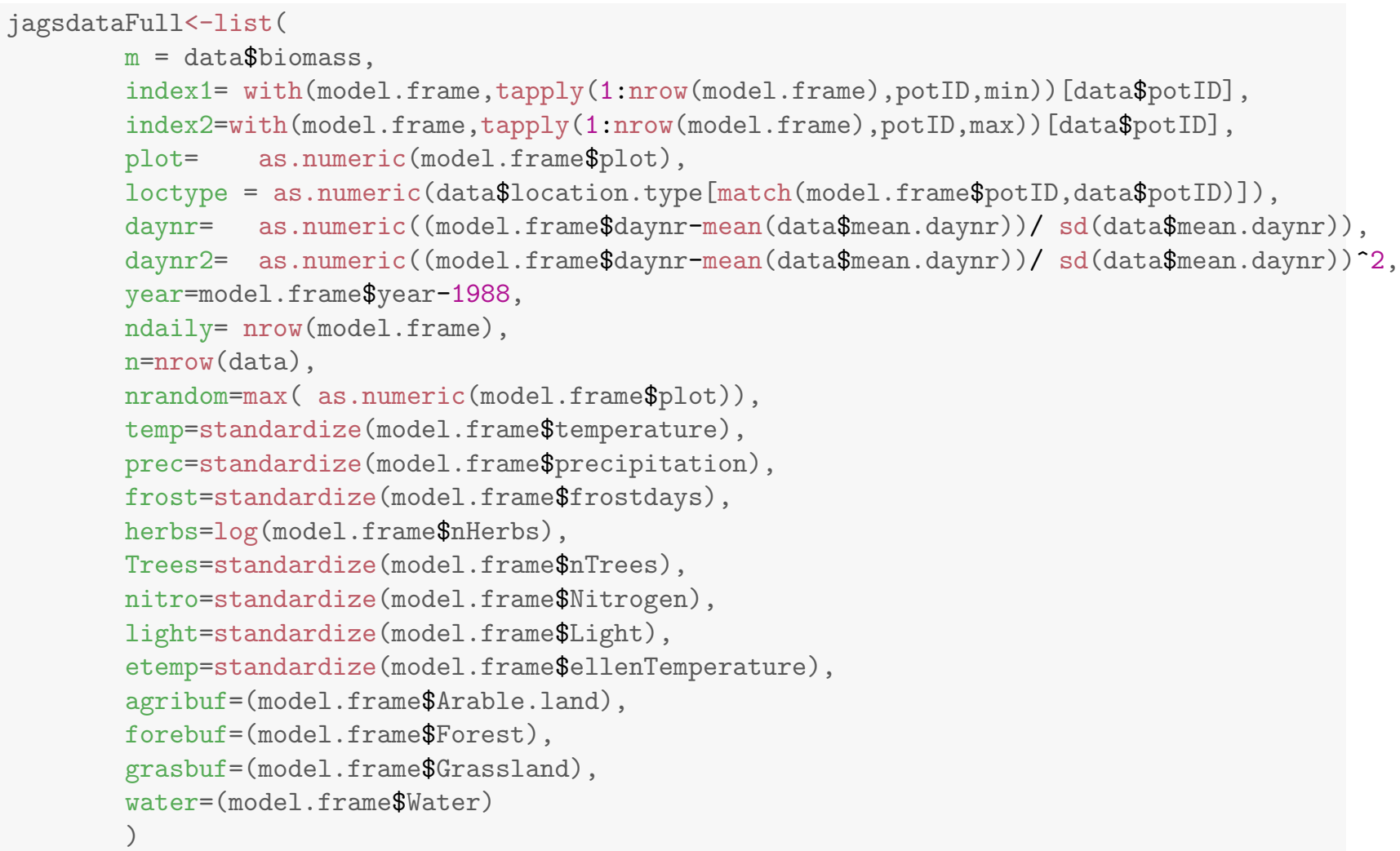

Write JAGS model file:

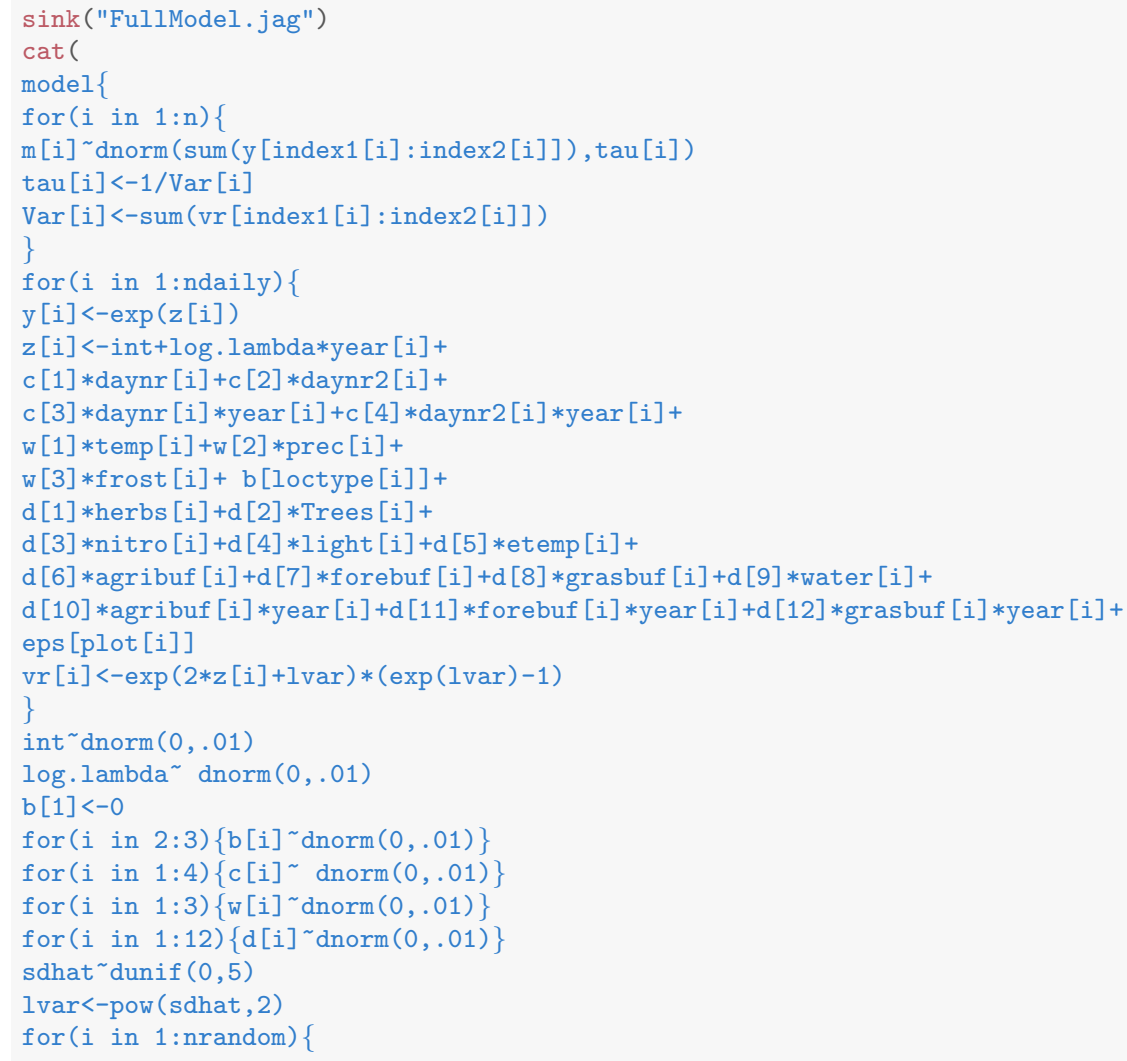




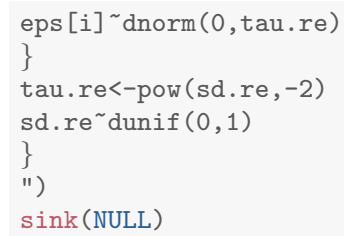

Run the model:

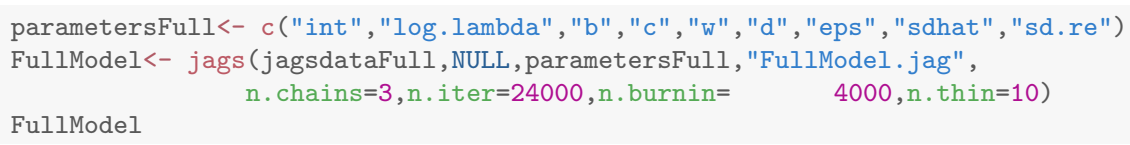

